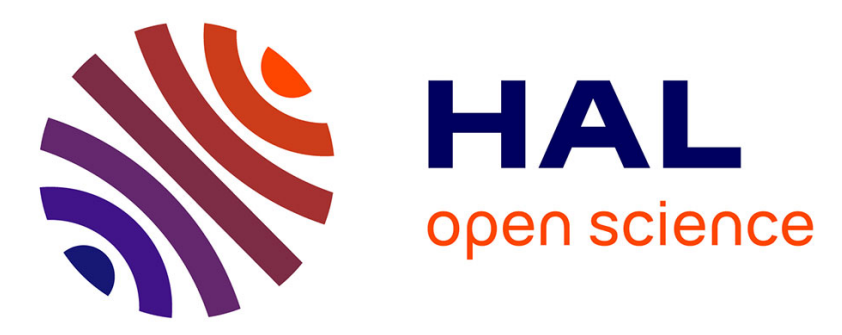

\title{
Superthermal-light emission and nontrivial photon statistics in small lasers
}

Tao Wang, Djeylan Aktas, O. Alibart, Eric Picholle, Gian Piero Puccioni, Sébastien Tanzilli, Gian Luca Lippi

\section{- To cite this version:}

Tao Wang, Djeylan Aktas, O. Alibart, Eric Picholle, Gian Piero Puccioni, et al.. Superthermal-light emission and nontrivial photon statistics in small lasers. Physical Review A, 2020, 101 (6), pp.063835. 10.1103/PhysRevA.101.063835 . hal-01614901

\section{HAL Id: hal-01614901 \\ https://hal.science/hal-01614901}

Submitted on 11 Oct 2017

HAL is a multi-disciplinary open access archive for the deposit and dissemination of scientific research documents, whether they are published or not. The documents may come from teaching and research institutions in France or abroad, or from public or private research centers.
L'archive ouverte pluridisciplinaire HAL, est destinée au dépôt et à la diffusion de documents scientifiques de niveau recherche, publiés ou non, émanant des établissements d'enseignement et de recherche français ou étrangers, des laboratoires publics ou privés. 


\title{
Nontrivial photon statistics in small scale lasers
}

\author{
T. Wang, ${ }^{1, \oplus, *}$ D. Aktas, ${ }^{1, \text { * }}$ O. Alibart,${ }^{1}$ É. Picholle, ${ }^{1}$ G.P. Puccioni, ${ }^{2}$ S. Tanzilli, ${ }^{1}$ and G.L. Lippi ${ }^{1}$ \\ ${ }^{1}$ Université Côte d'Azur, Institut de Physique de Nice (INPHYNI), CNRS UMR 7010, Nice, France \\ ${ }^{2}$ Istituto dei Sistemi Complessi, CNR, Via Madonna del Piano 10, I-50019 Sesto Fiorentino, Italy
}

(Dated: October 6, 2017)

\begin{abstract}
Photon statistical measurements on a semiconductor microlaser, obtained using single-photon counting techniques, show that a newly discovered pulsed emission regime is inconsistent with all current theoretical modeling. The observed spike dynamics, typical of small-scale devices, is at the origin of an unexpected discordance between the probability density function and its representation in terms of the first moments, a discordance so far unnoticed in all devices (even solid-state microcavities). The impact of this new dynamics is potentially large, since coincidence techniques are presently the sole capable of characterizing the light emitted by nanolasers.
\end{abstract}

Nanolasers are key components of future photonics integrated circuits [1, and their impact on the rapidly evolving field of information technology is already taking shape in the form of devices which promise ever faster transmission 2 24 and better integration levels. Their characterisation is based on the direct measurement of photon correlations which provide information on the coherence properties of the field even at very low photon flux levels [2 4]. This technique has been successfully used to discern the features of different microcavities [5], to study the lasing transition [6, 7], and as a proof of lasing for different designs $8-12$. In addition, techniques beyond two-detector correlation have been developed to improve both detection sensitivity and bandwidth [6, 13, 14. However, the interpretation of these measurements rests on a good understanding of the physics at the lasing transition.

In this paper, we show the existence of a correlation regime whose instrinsic features suggest strongly incoherent emission, but cannot be consistently interpreted using the two pillars of experimental photon statistics, namely: (1) the measurement of coincidence in the arrival times $\left(g^{(k)}(\tau)\right)$; and $(2)$ the measurement of Probability Density Function (PDF) $P(n)$ for the photon number $(n)$, reconstructing the most likely photon count for a given laser pump level, which requires linear detectors - a very serious challenge at extremely low signal levels.

In principle, the two techniques provide equivalent information as long as all moments $g^{(k)}$ of the distribution are measured. However, as experimental difficulties and costs grow with $k$, in most cases actual measurements are limited to $k=2$. In addition, the interpretation of available results for $k=3,4$ still remains difficult [15].

While the PDF for Class A lasers [16 is wellestablished, together with its connection to the secondorder moments [17, the memory effects introduced by the slow material response in Class B devices [16] require a more careful approach. Such a PDF has been established with suitable mathematical techniques [18, 19, extending early predictions valid unphysically close to threshold [20]. The experimental test of the PDF for Class B systems has confirmed its validity in a solid-state microcavity [21] - so-called extreme Class B device [21, 22] providing also the connection between the photon num- ber probability density function $(P(n))$ and the measured zero-delay second-order autocorrelation $\left(g^{(2)}(0)\right)$. The extensive work on solid-state microcavities was considered as a substitute for detailed measurements in semiconductor microdevices [23, technically impossible at the time. On the basis of the resounding confirmation of the validity of macroscopic Class B photon statistics in microcavities [18, 19] it was reasonably concluded [21] that its predictions should also hold for nanolasers.

The purpose of this paper is to reexamine this key issue by direct experimental comparison of $P(n)$ and $g^{(2)}(0)$ in a Vertical Cavity Surface Emitting Laser (VCSEL) with a moderate fraction of spontaneous emission coupled into the lasing mode $\left(\beta \approx 10^{-4}\right)$. The experiment consists of: (1) the measurement of $P(n)$, performed with a linear detector; and (2) the direct measurement of $g^{(2)}(0)$ by quantum interferometry. The full linear setup (24], SI) is summarized in Fig 1, top.

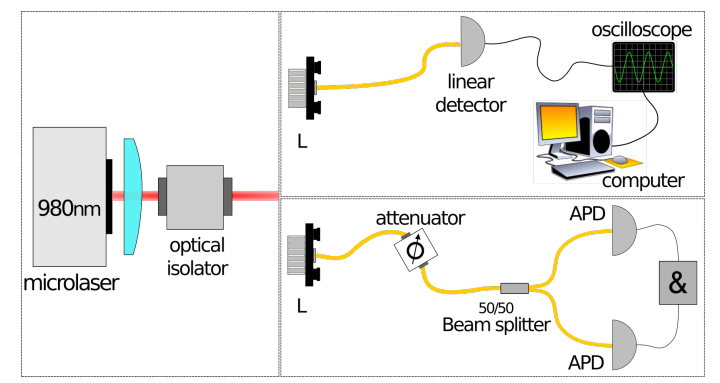

FIG. 1. Experimental setup. The laser (Thorlabs 980 VCSEL $-\lambda=980 \mathrm{~nm}-$, threshold current $i_{t h} \approx 1 \mathrm{~mA}$ ) is temperature-stabilized and supplied by a low-noise DC source (Thorlabs LDC200VCSEL). Top: The collimated laser output traverses an optical isolator, is collected by lens $\mathrm{L}$ and couples through a fiber into a $10 \mathrm{GHz}$ photodetector (Thorlabs PDA8GS). The signal, digitized by a LeCroy Wavemaster 8600 oscilloscope $\left(6 \mathrm{GHz}\right.$ analog bandwidth, $10^{6}$ points/trace), is stored for treatment. Bottom: The photon counting apparatus consists of an attenuator (Thorlabs VOA980 FC), a 50/50 fiber beamsplitter and 2 single photon detectors (APD idQuantique id100) with $\sim 40 \mathrm{ps}$ jitter. The AND gate $(\&)$ is made with a TAC (Ortec 567 ) with $\sim 15 \mathrm{ps}$ timing resolution.

Fig. 2 shows the histograms of the intensity distribution measured at significant values of pumping current 
(insets: time traces). The distributions show a distinct difference from Class A laser photon statistics, characterized by a large probability of recording no photons, and a similarity to those of extreme Class B devices [21, whose distributions start from the origin even at the lowest pump values (as in Fig. 2a).

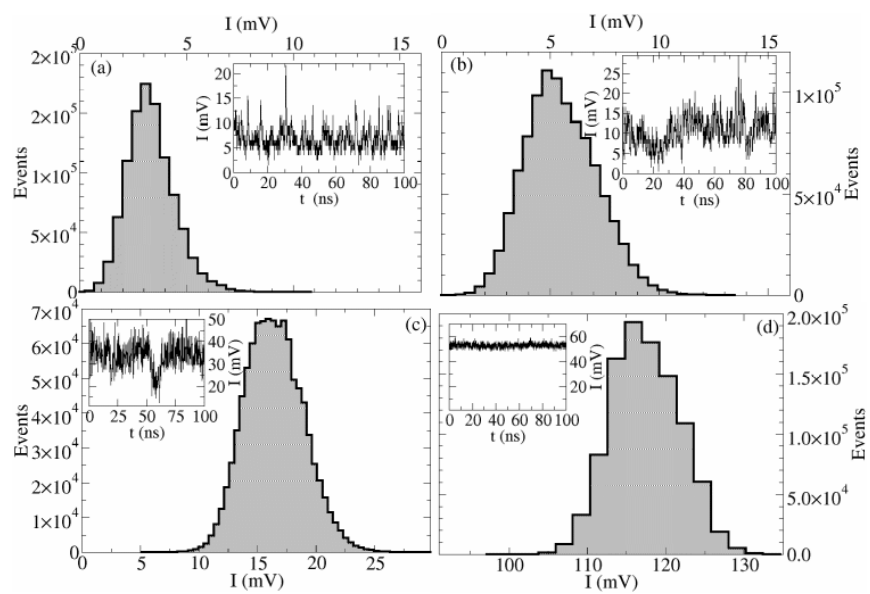

FIG. 2. Experimental PDF on $10^{6}$ points collected with the linear setup (Fig. 1. top). I represents the detected intensity (insets: temporal traces). Laser bias current: (a) $i=1.26 \mathrm{~mA}$ (beginning of laser emission); (b) $i=1.30 \mathrm{~mA}$ (laser pulses); (c) $i=1.45 \mathrm{~mA}$ (fully developed intensity oscillations); (d) $i=$ $3.0 \mathrm{~mA}$ (stable emission). The thick PDF outline highlights the long tails of the distribution.

Further, Fig. 2 provides interesting information on the shapes and sizes of the distributions. Panel (a), obtained closest to threshold, shows a narrow, peaked distribution with a tail towards large photon numbers (typical of a Class B laser PDF 18). A slight increase in the laser pump current quickly widens the distribution, still maintaining an asymmetric tail to the right (Fig. 2 $\mathrm{b}$ ). However, further increasing the pump current to reach a very noisy, but mostly continuous wave output (Fig. 2k), provides a much broader, nearly symmetric-looking distribution, analysed in the following. Finally, sufficiently far above threshold, we find the standard, low noise $(\lesssim 10 \%)$ above-threshold laser emission (Fig. 2d).

The measured statistical distributions (Fig. 2) are strongly affected by the data acquisition chain bandwidth $\left(2 \pi f_{B}<\frac{\Gamma_{c}}{10}, \Gamma_{c}\right.$ cavity relaxation rate). Since it is impossible to deconvolve its effect on the measured statistical distribution, we ressort to comparing our results to the photon number distribution numerically predicted by a Stochastic Simulator (SS) 25], shown to provide reliable predictions of the laser's dynamics 24. Comparison of the predicted statistics with and without the detection's filtering action offers a pertinent tool for bridging the gap between experimental observations and theoretical predictions [18. This test is performed on the distribution of Fig. 22, which corresponds to the very noisy laser output. The predictions of the SS are extracted from a large data sample $\left(10^{7}\right.$ points), with time binning short compared to the photon lifetime $\left(t_{b} \approx \frac{\Gamma_{c}^{-1}}{10}\right)$ to obtain the probability distribution represented by the dash-dotted line curve in Fig. 3. We then mimick the action of the detector by binning the data on a time $t_{b} \approx f_{B}^{-1}$ (dashed curve). The influence of the acquisition chain on $P(n)$ is dramatic: the distribution is strongly narrowed, and thus raised, with its maximum at considerable lower average photon numbers. The figures display the statistics of output photons - as in the experiment - rather than the usually predicted intracavity photon distribution. Comparison to the experimental distribution (filled histogram in Fig. 3) shows a good qualitative agreement between predicted and measured $P(n)$. The numerical curves have been rescaled to account for the detector's sensitivity and the horizontal scale is given in $\mathrm{mV}$ to preserve the experimental units.

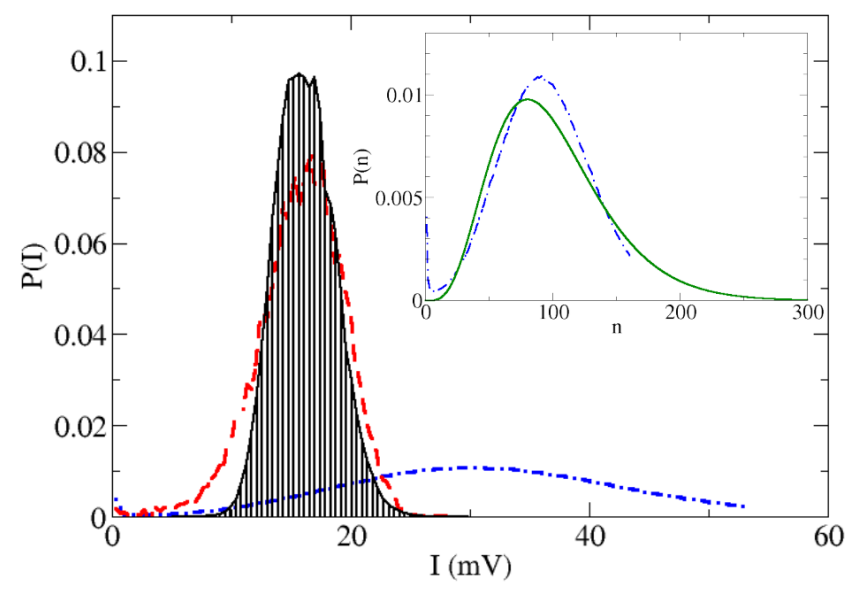

FIG. 3. Comparison between experimental distribution (hashed histogram, from Fig. 22) and numerical (SS) simulations to test the detector's filtering action. The solid line (red online) represents the filtered, the dot-dashed (blue online) the unfiltered numerical PDFs. Inset: Comparison between the theoretical $P(n)$ (solid line-green online) obtained from [18] and its numerical (SS) counterpart (dot-dashed line - blue online). The accumulation of events in the " 0 " channel in the simulation is due to the absence of external noise, which results in the appearence of strictly zero photons after an occasional very large fluctuation.

Matching the experiment to the theoretical probability distribution requires an additional step. The inset in Fig. 3 reproduces the computed $P(n)$ (same dot-dashed curve as in the main figure) impinging on an ideal, nonbandwidth-limited detector, plotted together with the theoretical intracavity photon distribution [18, suitably rescaled to take into account for the output coupler transmission (solid line, green online). The values for the two parameters characterizing the probability distribution are chosen to be $\bar{n}=3 \times 10^{4}$ [26], and $g^{(2)}(0)=1.2$, inferred from the zero-delay second-order autocorrelation measurements 24], where we have taken into account the correction due to the detection bandwidth which reduces $g^{(2)}(0)$ by approximately one order of magnitude.

The numerical histogram is sharply truncated (inset of Fig. 3p. This cutoff comes from the limited number of 
emitters $\left(\approx 10^{5}\right.$ for a multiple quantum well VCSEL $[27)$ and from the small amount of fluctuations in the carrier density tolerated around threshold: $\sim 1 \%$ is sufficient to turn off the laser. In a solid-state microcavity the photon number fluctuations supported by the laser are much larger due to low output coupler reflectivity $(R=0.8)$ 222, whence the much longer tails in the experimental PDF 21 .

Overall, considering that some parameters, such as cavity losses and detection efficiency, can only be estimated with a large error margin, the match is quite satisfactory. We thus conclude, by comparing the curves in the main part and inset of Fig. 3. that the experimental photon distribution is compatible with the predictions of theoretical models for Class B lasers [18, and confers them generality. However, this infirms, together with Fig. 2 , the conclusion based on the criterion 21 that lasers with $\beta \Lambda<1\left(\Lambda=\frac{\Gamma_{c}}{\gamma_{\|}}[22,28], \gamma_{\|}\right.$carrier relaxation rate $)$are weakly Class $B$ and follow standard (i.e., Class A) photon statistics. The present measurements, enabled by modern instrumentation, shed a new light onto the photon statistics of semiconductor microcavities.

Scaling down the cavity size requires, however, a different approach and we therefore turn to comparing the results on the PDF to those coming from a Hanbury-Brown $\&$ Twiss (HBT) experiment in the single photon counting regime, whose sensitivity can reach the femtowatt level. In addition to allowing detection at much lower power, far below threshold in our case, this technique increases the effective measurement bandwidth. The detection jitter $(\sim 40 \mathrm{ps})$ associated with a time-digitizer resolution of $\sim$ $15 \mathrm{ps}$ leads to a coincidence detection equivalent bandwith of $17 \mathrm{GHz}$. The combination of these factors enhances the fidelity in the reproduction of the laser's behaviour and, through the direct comparison between $P(n)$ and $g^{(2)}(0)$, sheds additional light on what is to be expected with nanolasers.

The autocorrelation function is obtained through coincidence measurements using two single photon detectors (Fig. 1. bottom), recording with a time-to-amplitudeconverter the time distribution of coincidences between the two detectors for different values of the injection current. Suitably normalized, this distribution is directly related to the autocorrelation function $g^{(2)}(\tau)$. We assume that, in a given integration time window $\Delta T$, the detectors register $N_{t}$ photons in the beamsplitter transmission and $N_{r}$ photons in reflection. Proper normalization is obtained by assuming that for long time delays $g^{(2)}(\infty) \rightarrow 1$. We therefore calculate the second order autocorrelation function, $g^{(2)}(\tau)=N_{c} / N_{t} N_{r}\left(\frac{\Delta T}{\Delta t}\right)$, where $N_{c}$ is the number of coincidence detection per time bin $\Delta t$, i.e., the resolution of the system used for coincidence measurement.

Fig. 4 summarizes the experimental measurement of the zero-delay second-order autocorrelation as a function of the laser injection current, compared to the linear measurement 24]. The two curves are in reasonable qualitative agreement, since the strong bandwidth limi-

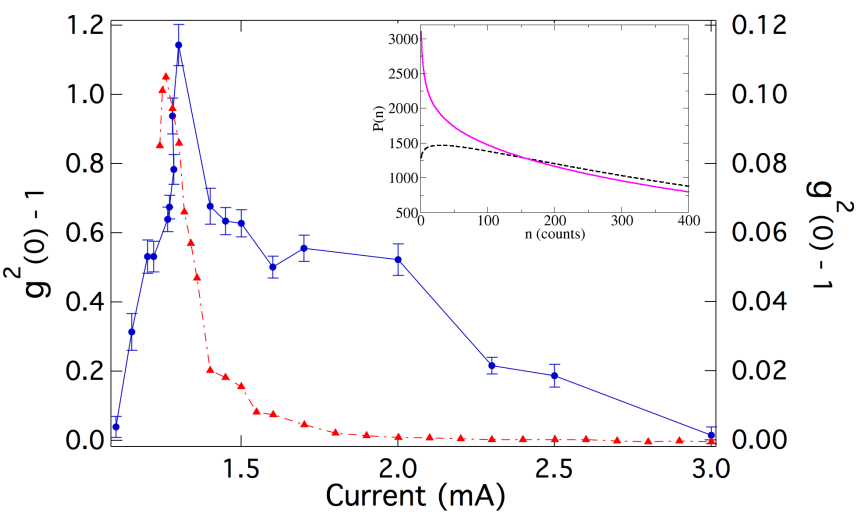

FIG. 4. Scaled second-order autocorrelation $\left(g^{(2)}(\tau=0)\right)$ obtained with the HBT setup as a function of the injection current (dots - blue online - left vertical scale). For comparison, the previously reported measurements of $g^{(2)}(\tau=0)$ 24 obtained from linear detection (triangles - red online right vertical scale). Inset: Photon number PDF predicted by the Class B model (eqs. (23p) at the autocorrelation peak: $g_{0}^{(2)}=2.15$ (solid line, magenta online). For comparison, the PDF predicted shape for $g_{0}^{(2)}=1.95<2$ (dashed line, black online).

tation reduces $g_{\text {lin }}^{(2)}(0) \approx \frac{g^{(2)}(0)}{10}[24$. The peaks of the two curves are nearly coincident: the slight displacement is consistent with different laboratory temperature stabilization. The drop for low values of current (left of peak) is due to the residual bandwith limitation, while the steep drop from the peak (to its right) appears in both sets of measurements. Two plateaus $((1.4 \lesssim i \lesssim 1.6) \mathrm{mA}$ and $(1.7 \lesssim i \lesssim 2.0) \mathrm{mA})$ appear in both cases, with the second one (top curve) being relatively higher than the one obtained in the linear measurement. We attribute this quantitative discrepancy to the high sensitivity of the laser to any kind of perturbation in this range and to the much higher sensitivity of the HBT setup. For $i>2.0 \mathrm{~mA}$ the autocorrelation drops towards the Poisson limit, reached only at $i \approx 4.5 \mathrm{~mA}$, rather than at $i \approx 3.0 \mathrm{~mA}$ as previously indicated by the less sensitive linear technique [24].

Globally, the two independent measurements support each other, with the HBT-based autocorrelation allowing for a more quantitatively dependable estimate, thanks to its higher performance and simple self-calibration. The expected standard value for the spontaneous emission regime $\left(g^{(2)}(0)=2\right)$ is not observed, due to the temporal resolution of the coincidence measurement, but the maximum of $g^{(2)}(0)$ overcomes, within its error bar, the $g^{(2)}(0)=2$ barrier. While several physical mechanisms can give rise to such effects, such as quantum interference [29], none of them are likely to intervene in our experiment. Instead, the following example shows that the pulsing dynamics 24] which correspond to the peak in $g^{(2)}(0)$ (Fig. 2a), may explain its anomalously large value.

Consider an asymmetric square wave of period $T$, emulating pulses, with high and low levels at $A$ and $\alpha A$ and 
durations $T \delta$ and $T(1-\delta)$, respectively. Its $g^{(2)}(0)$ reads:

$$
g^{(2)}(0)=\frac{\frac{1}{N}\left[N \delta A^{2}+N(1-\delta) \alpha^{2} A^{2}\right]}{\frac{1}{N^{2}}[N \delta A+N(1-\delta) \alpha A]^{2}} \approx \frac{1}{\delta(1+c)^{2}},(1)
$$

where the last expression is obtained, to leading order, for narrow pulses $(\delta \rightarrow 0)$, after rewriting, for convenience, $\alpha=c \delta(c$ being an arbitrary constant $O(1))$. This shows that $g^{(2)}(0)$ grows without bounds as $\delta \ll 1$ (independently of $c$ ). Hence, it is not surprising that for finite-size pulses $g^{(2)}(0)$ may take values larger than those expected for a chaotic signal [15] and we can use it as an indicator of the laser spiking observed in 24].

The formulation of Class-B photon statistics [18, 19. in terms of average photon number, $n_{a}$, and $g^{(2)}(0)[21]$ allows for matching the statistical distributions (Fig. 2) to the autocorrelation measurements (Fig. 4):

$$
\begin{aligned}
P(n) & =C\left(n_{a}, g_{2,0}\right) n^{\frac{2-g_{2,0}}{g_{2,0}-1}} e^{-\frac{n}{n_{a}\left[g_{2,0}-1\right]}} \\
C\left(n_{a}, g_{2,0}\right) & =\frac{1}{\Gamma\left(\frac{1}{g_{2,0}-1}\right)}\left(\frac{1}{n_{a}\left(g_{2,0}-1\right)}\right)^{\frac{1}{g_{2,0}-1}},
\end{aligned}
$$

where we have explicitely written the coefficients given in [21] and used the shorthand $g_{2,0}$ in place of $g^{(2)}(0)$. Inspection shows immediately that the nature of the distribution changes entirely when $g^{(2)}(0)>2$ and transforms the PDF into a monotonically decreasing distribution, as shown in the inset of Fig. 4 (solid line), which is clearly incompatible with our experimental observations (Fig. 2a), as well as with the expected generalization of previous results [21. The apparent lack of bijectivity between the two sets of data signals an underlying limitation of the model [18, 19] which, by excluding the contribution of spontaneous emission, appears to be intrinsically unable to predict the occurrence of the dynamics observed in Fig. 2a,b. Since the PDF collects into a statistical distribution the state of the system, its predictions fail when the dynamics cannot be reproduced by the model from which it is derived. While we report here the spiking dynamics on one type of device, we have observed it on several VCSELs, from different manufacturers and with different sizes (up to $\beta \approx 10^{-3}$ ). Similar spiking has been observed and reported in the past on VCSELs (see, e.g. 30]), but has typically been ascribed either to competition between polarization modes close to threshold or to technical noise. Some of us have already shown that this dynamics is intrinsic to the system and we prove here that it possesses nontrivial physical features [24].

The consequences of this realization are far reaching. Paraphrasing Lien et al. 21], the non-standard Class-B photon statistics, which unexpectedly matched the experimental observations, was thought to hold for lasers with $\Lambda \beta \gtrsim 1$, as proven on a microcavity solid state laser, while for semiconductor devices it was thought that one should reach at least $\Lambda \beta \gtrsim 0.1$ in order to see the first deviations from standard Class-A photon statistics 21. Our current results show that already for $\Lambda \beta \lesssim 10^{-2}$ Class-B photon statistics [18, 19] are in fair agreement with the experimental observations. Furthermore, we find an important deviation from the predicted - and so far observed - photon statistics in the threshold region. Deviations from standard photon statistics were expected close to threshold, but were thought to lead towards the distributions which had been experimentally validated in solid state microcavities 21. Thus the question naturally arises as to why the dynamics of Fig. $2 \mathrm{a}$ were not previously observed 23. The most likely reason is that in spite of the relatively large $\beta$ value achieved for a solid state microcavity $\left(\beta \approx 10^{-5}\right)$, its volume remained too large to provide a sufficiently broad parameter region (pump rate range) to experimentally access the pulsing regime. As the steepness of the laser S-shaped response curve sensitively depends on $\beta^{-1}$, the range of pump rate values in which such dynamics can be observed shrinks. In addition, the extreme Class $B$ nature of the solid state microcavity 21 renders the observation of such dynamics even harder since the strong imbalance between cavity losses and population inversion relaxation rate makes the device more sensitive to pump fluctuations. The regime of strong oscillations (cf. Fig. 2k) was indeed reported 23, but no spiking dynamics appears to have been observed. This explains the inadequacy between the conclusions which could be drawn [21 23] and our current observations.

One important consequence of this discovery lies in the interpretation of the measurements of $g^{(2)}(0)$, a crucial step in the characterization of nanolasers, since for the latter it is impossible to obtain the full information possible with our larger devices. Our experiment, conducted in a $\beta \approx 10^{-4}$ device, represents a happy compromise between (large enough) cavity size to observe the dynamics, and a clear set of well-isolated spikes (which require a low enough $\beta$ - cf. Fig. 5 in the Supplementary Information of 24). For larger values of $\beta$ we do not observe $g^{(2)}(0)>2$ and would miss the clear warning which comes from the inconsistency signalled by the comparison between the predictions of Fig. 4 and the observations of Fig. 2a. Nonetheless, the inconsistent description of the statistics remains since the spiking dynamical regime - incompatible with all currently known forms of photon statistics - exists in all small lasers down to the nanoscale (see, e.g. [7, 12]) and would distort the interpretation of the analysis of the observed autocorrelation $\left(g^{(2)}(0)\right)$ functions.

Therefore we conclude that new theoretical work is needed on the photon statistics of small-sized Class B lasers, since the current available models are based on an incomplete description of the threshold region, which extends over an ever larger range of pump rate values as $\beta$ increases. Indeed, since next-generation photonics integrated circuits can be expected to be based on nanolasers operating in the few-photon regime, a good understanding of their photon statistics might prove a significant technological issue in the near future.

Partial funding was obtained from the Fédération W. 
Döblin. GLL acknowledges enlightening discussions with A. Politi. T.W. is grateful to the Conseil Régional PACA for funding and to BBright for support.

$\dagger$ Currently at: School of Physics and Electronic Science, Hunan University of Science and Technology, Xiangtan 411201, China

* These Authors have equally contributed to the work

[1] M. Smit, J. van der Tol, and M. Hill, Laser \& Photonics Review 6, 1 (2012).

[2] B. Mayer, L. Janker, B. Loitsch, J. Treu, T. Kostenbader, S. Lichtmannecker, T. Reichert, S. Morkötter, M. Kaniber, G. Abstreiter, C. Gies, G. Koblmüller, and J.J. Finley, Nano Letters 16, 152 (2015).

[3] A. Yokoo, M. Takiguchi, M.D.Birowosuto, K. Tateno, G. Zhang, E. Kuramochi, A. Shinya, H. Taniyama, and M. Notomi, ACS Photonics 4, 355 (2017).

[4] M. Takiguchi, A. Yokoo, K. Nozaki, M.D. Birowosuto, K. Tateno, G. Zhang, E. Kuramochi, A. Shinya, and M. Notomi, APL Photonics 2, 046106 (2017).

[5] S.M. Ulrich, C. Gies, S. Ates, J. Wiersig, S. Reitzenstein, C. Hofmann, A. Löffler, A. Forchel, F. Jahnke, and P. Michler, Phys. Rev. Lett. 98, 043906 (2007).

[6] N. Takemura, J. Omachi, and M. Kuwata-Gonokami, Phys. Rev. A 85, 053811 (2012).

[7] A. Lebreton, I. Abram, R. Braive, I. Sagnes, I. RobertPhilip, and A. Beveratos, Phys. Rev. Lett. 110, 163603 (2013).

[8] S. Strauf, K. Hennessy, M. T. Rakher, Y.-S. Choi, A. Badolato, L. C. Andreani, E. L. Hu, P. M. Petroff, and D. Bouwmeester, Phys. Rev. Lett. 96, 127404 (2006).

[9] R. Hostein, R. Braive, L. Le Gratiet, A. Talneau, G. Beaudoin, I. Robert-Philip, I. Sagnes, and A. Beveratos , Opt. Lett. 35, 1154 (2010).

[10] M. Takiguchi, H. Taniyama, H. Sumikura, M. D. Birowosuto, E. Kuramochi, A. Shinya, T. Sato, K. Takeda, S. Matsuo, and M. Notomi, Opt. Express 24, 3441 (2015).

[11] W.E. Hayenga, H. Garcia-Gracia, H. Hodaei, Ch. Reimer, R. Morandotti, P. Likamwa, and M. Khajavikhan, Optica 3, 1187 (2016).

[12] S.H. Pan, Q. Gu, A. El Amili, F. Vallini, and Y. Fainman, Optica 3, 1260 (2016).

[13] J. Wiersig, C. Gies, F. Jahnke, M. Aßmann, T. Berstermann, M. Bayer, C. Kistner, S. Reitzenstein, C. Schnei- der, S. Höfling, A. Forchel, C. Kruse, J. Kalden, and D. Hommel, Nature 460, 245 (2009).

[14] Y. Ota, K. Watanabe, S. Iwamoto, and Y. Arakawa, Phys. Rev. A 89, 023824 (2014).

[15] D. Elvira, X. Hachair, V.B. Verma, R. Braive, G. Beaudoin, I. Robert-Philip, I. Sagnes, B. Baek, S. W. Nam, E.A. Dauler, I. Abram, M.J. Stevens, and A. Beveratos, Phys. Rev. A 84, 061802(R) (2011).

[16] J.R. Tredicce, F.T. Arecchi, G.L. Lippi, and G.P. Puccioni, J. Opt. Soc. Am. B2, 173 (1985).

[17] F.T. Arecchi and V. Degiorgio, Phys. Rev. A 3, 1108 (1971).

[18] P. Paoli, A. Politi, and F.T. Arecchi, Z. Physik B 71, 403 (1988).

[19] T. Ogawa, Phys. Rev. A 42, 4210 (1990).

[20] H. Risken, Z. Physik 186, 85 (1965).

[21] Y. Lien, S.M. de Vries, N.J. van Druten, M.P. van Exter, and J.P. Woerdman, Phys. Rev. Lett. 86, 2786 (2001).

[22] N.J. van Druten, Y. Lien, C. Serrat, S.S.R. Oemrawsingh, M.P. van Exter, and J.P. Woerdman, Phys. Rev. A 62, 053808 (2000).

[23] J.P. Woerdman, M.P. Van Exter, and N.J. Van Druten, Adv. At. Mol. Opt. Phys. 47, 205 (2001).

[24] T. Wang, G.P. Puccioni, and G.L. Lippi, Sci. Rep. 5, 15858 (2015).

[25] G.P. Puccioni and G.L. Lippi, Opt. Express 23, 2369 (2015)

[26] This value is estimated from $\bar{n} \approx \frac{N_{p h} \times n_{M}}{b \times R}$, where $b \approx$ 0.05 is the effective bandwidth - ratio of the acquisition system's response rate $\Gamma_{a}$ in units of the photon's cavity lifetime $\Gamma_{c} ; N_{p h}-\left(N_{p h} \approx 150 m V^{-1}\right.$, cf. Thorlabs' specifications for the PDA8GS photodetector) - is the expected number of detected photons per unit of measurement; $n_{M} \approx 17 \mathrm{mV}$ is the peak of the experimental photon distribution (Fig. 2 2 ), and $R \approx 0.425 \%$ is an estimate for the outcoupled photon fraction.

[27] Considering a 4 Quantum Well (QW) VCSEL with effective pumped surface $s_{\text {eff }} \approx 3 \times 10^{-7} \mathrm{~cm}^{2}, 80 \mathrm{~nm} \mathrm{QW}$ thickness and electron density $2 \times 10^{18} \mathrm{~cm}^{-3}$, we obtain a carrier number $\approx 2 \times 10^{5}$, compatible with the numerics of the SS which operates with $N \approx 4 \times 10^{5}$.

[28] H.F. Hofmann and O. Hess, Phys. Rev. A 62, 063807 (2000).

[29] F. Jahnke, Ch. Gies, M. Aßmann, M. Bayer, H.A.M. Leymann, A. Foerster, J. Wiersig, Ch. Schneider, M. Kamp, and S. Höfling, Nature Comm. 7, 11540 (2016).

[30] M. Sondermann, M. Weinkath, T. Ackemann, J. Mulet, and S. Balle, Phys. Rev. A 68, 033822 (2003). 